\title{
On Cluster Points, Continuity, and Boundedness Associated with the Generalized Statistical Convergence in Probabilistic Normed Spaces
}

\author{
Pratulananda Das, ${ }^{1}$ Kaustubh Dutta, ${ }^{1}$ and Vatan Karakaya ${ }^{2}$ \\ ${ }^{1}$ Department of Mathematics, Jadavpur University, Jadavpur, Kolkata, West Bengal 700032, India \\ ${ }^{2}$ Department of Mathematical Engineering, Yildiz Technical University, Esenler, 34210 Istanbul, Turkey \\ Correspondence should be addressed to Vatan Karakaya; vkkaya@yildiz.edu.tr
}

Received 26 October 2013; Accepted 6 April 2014; Published 28 April 2014

Academic Editor: Csaba Varga

Copyright (C) 2014 Pratulananda Das et al. This is an open access article distributed under the Creative Commons Attribution License, which permits unrestricted use, distribution, and reproduction in any medium, provided the original work is properly cited.

We consider the recently introduced notion of $\mathscr{I}$-statistical convergence (Das, Savas and Ghosal, Appl. Math. Lett., 24(9) (2011), 1509-1514, Savas and Das, Appl. Math. Lett. 24(6) (2011), 826-830) in probabilistic normed spaces and in the following (Şençimen and Pehlivan $(2008$ vol. 26,2008 vol. 87, 2009)) we introduce the notions like strong $\mathscr{I}$-statistical cluster points and extremal limit points, and strong $\mathscr{I}$-statistical continuity and strong $\mathscr{I}$-statistical $D$-boundedness in probabilistic normed spaces and study some of their important properties.

\section{Introduction}

The idea of convergence of real sequences had been extended to statistical convergence by Fast [1] and basic ideas were further developed in [2-5]. Recall that "asymptotic density" of a set $A \subseteq \mathbb{N}$ is defined as

$$
d(A)=\lim _{n \rightarrow \infty} \frac{1}{n}|\{k \leq n: k \in A\}|,
$$

provided that the limit exists, where $\mathbb{N}$ denotes the set of natural numbers and the vertical bar stands for cardinality of the enclosed set. The sequence $\left\{p_{n}\right\}_{n \in \mathbb{N}}$ of reals is said to be statistically convergent to a real number $p$ if, for each $\epsilon>0$,

$$
\lim _{n \rightarrow \infty} \frac{1}{n}\left|\left\{k \leq n:\left|x_{k}-x\right| \geq \epsilon\right\}\right|=0 .
$$

The concepts of $\mathscr{I}$ and $\mathscr{I}^{*}$-convergence, two important generalizations of statistical convergence, were introduced and investigated by Kostyrko et al. [6]. The ideas were based on the notion of ideal $\mathscr{I}$ of $\mathbb{N}$. Subsequently, a lot of investigations have been done on ideal convergence (see [717] where many more references both on ideal as well as statistical convergence can be found). Very recently, ideals were used in a different way to generalize the notion of statistical convergence $[18,19]$ and certain new and summability methods were introduced and their basic properties were investigated. More recently these ideas were extended to double sequences in [20].

On the other hand, the idea of probabilistic metric space was first introduced by Menger [21] in the name of "statistical metric space." Probabilistic normed space (briefly PN space) is a generalisation of an ordinary normed linear space. In a PN space, the norms of the vectors are represented by the distribution functions instead of nonnegative real numbers. Detailed theory of these spaces can be found in the famous book written by Schweizer and Sklar [22] and the monogram [23]. One can also see the papers [22, 24-35] where the basic ideas were established. Several topologies can be defined on this space. But the topology that was found to be most useful is the "strong topology." Şençimen and Pehlivan have very recently extended the notion of strong convergence to strong statistical convergence in probabilistic metric spaces [36] and carried out further investigations on statistical continuity and statistical $D$-boundedness in PN spaces $[37,38]$. These were followed by the studies of strong ideal convergence in PM and PN spaces in $[10,13,39]$, studies of lacunary statistical 
convergence in PN spaces in [40]. As a natural extension, we had recently introduced the idea of strong $\mathscr{I}$-statistical convergence in PM spaces [41] and as a followup in this paper we investigate the notion of strong $\mathscr{I}$-statistical limit and cluster points in PN spaces. Further, we have introduced the concepts like strong $\mathscr{I}$-statistical continuity and strong $\mathscr{I}$-statistical $D$-boundedness in such spaces and investigated some of their important properties.

\section{Preliminaries}

First, we recall some of the basic concepts related to the theory of probabilistic metric and normed spaces (see [22, 23, 3139, 42] for more details).

Definition 1. A nondecreasing function $F: \mathbb{R} \rightarrow[0,1]$ defined on $\mathbb{R}$ with $F(-\infty)=0$ and $F(\infty)=1$, where $\mathbb{R}=$ $[-\infty, \infty]$, is called a distribution function.

The set of all left continuous distribution functions over $(-\infty, \infty)$ is denoted by $\Delta$. One considers the relation " $\leq$ " on $\Delta$ defined by $F \leq G$ if and only if $F(x) \leq G(x)$ for all $x \in \mathbb{R}$. It can be easily verified that the relation " $\leq$ " is a partially order on $\Delta$.

Definition 2. For any $a \in \mathbb{R}$, the unit step function at $a$ is denoted by $\varepsilon_{a}$ and is defined to be a function in $\Delta$ given by

$$
\varepsilon_{a}(x)= \begin{cases}0, & \text { if }-\infty \leq x \leq a, \\ 1, & \text { if } a<x \leq \infty .\end{cases}
$$

Definition 3. A sequence $\left\{F_{n}\right\}_{n \in \mathbb{N}}$ of distribution functions converges weakly to a distribution function $F$ and one writes $F_{n} \stackrel{w}{\rightarrow} F$ if and only if the sequence $\left\{F_{n}(x)\right\}_{n \in \mathbb{N}}$ converges to $F(x)$ at each continuity point $x$ of $F$.

Definition 4. The distance between $F$ and $G$ in $\Delta$ is denoted by $d_{L}(F, G)$ and is defined as the infimum of all numbers $h \epsilon$ $(0,1]$ such that the inequalities

$$
\begin{aligned}
& F(x-h)-h \leq G(x) \leq F(x+h)+h, \\
& G(x-h)-h \leq F(x) \leq G(x+h)+h
\end{aligned}
$$

hold for every $x \in(-1 / h, 1 / h)$.

Here, we are interested in the subset of $\Delta$ consisting of those elements $F$ that satisfy $F(0)=0$.

Definition 5. A distance distribution function is a nondecreasing function $F$ defined on $\mathbb{R}^{+}=[0, \infty]$ that satisfies $F(0)=0$ and $F(\infty)=1$ and is left continuous on $(0, \infty)$.

The set of all distance distribution functions is denoted by $\Delta^{+}$. The function $d_{L}$ is clearly a metric on $\Delta^{+}$. The metric space $\left(\Delta^{+}, d_{L}\right)$ is compact and hence complete.

Theorem 6. Let $F \in \Delta^{+}$be given. Then, for any $t>0, F(t)>$ $1-t$ if and only if $d_{L}\left(F, \varepsilon_{0}\right)<t$.

Note. Geometrically, $d_{L}\left(F, \varepsilon_{0}\right)$ is the abscissa of the point of intersection of the line $y=1-x$ and the graph of $F$ (if necessary we add vertical line segment at the point of discontinuity).

Definition 7. A triangular norm (briefly, a $t$-norm) $T$ is a binary operation on the unit interval $T:[0,1] \times[0,1] \rightarrow$ $[0,1]$ that is associative, commutative, nondecreasing in each place, and has 1 as identity. The operations defined by $M(x, y)=\min \{x, y\}$ and $\pi(x, y)=x y$ are particular $t$ norms. Given a $t$-norm $T$, its $T$-conorm $T^{*}$ is defined as a mapping on $[0,1] \times[0,1]$ by $T^{*}(x, y)=1-T(1-x, 1-y)$.

Definition 8. A triangle function is a binary operation $\tau$ on $\Delta^{+}, \tau: \Delta^{+} \times \Delta^{+} \rightarrow \Delta^{+}$, which is commutative, associative, and nondecreasing in each place, and has $\varepsilon_{0}$ as identity. Triangle functions can be constructed through leftcontinuous $t$-norms. If $T$ is such a $T$-norm, then

$$
\tau_{T}(F, G)(x)=\sup \{T(F(s), G(t)): s+t=x\}
$$

is a triangle function, where $x \in \mathbb{R}^{+}$. If, moreover, $T$ is continuous, then $\tau_{T}$ is uniformly continuous on $\left(\Delta^{+}, d_{L}\right)$. If $T^{*}$ is a continuous $t$-conorm, then

$$
\tau_{T^{*}}(F, G)(x)=\inf \left\{T^{*}(F(s), G(t)): s+t=x\right\}
$$

is a triangle function which is uniformly continuous on $\left(\Delta^{+}, d_{L}\right)$.

Definition 9. A probabilistic metric space (briefly a PM space) is a triplet $(X, \mathfrak{F}, \tau)$ where $X$ is a nonempty set, $\mathfrak{F}$ is a function from $X \times X$ into $\Delta^{+}$, and $\tau$ is a triangle function. The following conditions are satisfied for all $x, y, z \in X$ :

(PM1) $\mathfrak{F}(x, x)=\varepsilon_{0}$;

(PM2) $\mathfrak{F}(x, y) \neq \varepsilon_{0}$ if $x \neq y$;

(PM3) $\mathfrak{F}(x, y)=\mathfrak{F}(y, x)$;

(PM4) $\mathfrak{F}(x, z) \geq \tau(\mathfrak{F}(x, y), \mathfrak{F}(y, z))$.

In the sequel, we will denote $\mathfrak{F}(x, y)$ by $F_{x y}$ and its value at $t$ by $F_{x y}(t)$.

Definition 10. A probabilistic normed space (briefly a PN space $)$ is a quadruple $\left(X, \eta, \tau, \tau^{*}\right)$, where $X$ is a real linear space, $\tau$ and $\tau^{*}$ are continuous triangle functions with $\tau \leq \tau^{*}$, and $\eta$ is a mapping (the probabilistic norm) from $X$ into the space of distribution functions $\Delta^{+}$such that, writing $N_{p}$ for $\eta(p)$ for all $p, q$ in $X$, the following conditions hold:

(N1) $N_{p}=\varepsilon_{0}$ if and only if $p=\theta$, the null vector in $X$;

(N2) $N_{-p}=N_{p}$;

(N3) $N_{p+q} \geq \tau\left(N_{p}, N_{q}\right)$;

(N4) $N_{p} \leq \tau^{*}\left(N_{\alpha p}, N_{(1-\alpha) p}\right)$ for all $\alpha \in[0,1]$.

A Menger PN space under $T$ is a $\mathrm{PN}$ space $\left(X, \eta, \tau, \tau^{*}\right)$ in which $\tau=\tau_{T}$ and $\tau^{*}=\tau_{T^{*}}$ for some continuous $t$-norm $T$ and its $t$-conorm $T^{*}$. It is denoted by $(X, \eta, T)$.

Throughout the text, $X$ will represent the PN space $\left(X, \eta, \tau, \tau^{*}\right)$. 
Theorem 11. Let $\left(X, \eta, \tau, \tau^{*}\right)$ be a probabilistic normed space and let $\mathfrak{F}$ be the function from $X \times X$ to $\Delta^{+}$defined by

$$
\mathfrak{F}(x, y)=\eta(x-y)=N_{x-y} .
$$

Then, $\left(X, \eta, \tau, \tau^{*}\right)$ is a probabilistic metric space (briefly PM space).

Definition 12. Let $X$ be a PN space. For $x \in X$ and $t>0$, the strong $t$-neighbourhood of $p$ is defined as the set

$$
\mathcal{N}_{p}(t)=\left\{q \in X: d_{L}\left(N_{p-q}, \varepsilon_{0}\right)<t\right\} .
$$

Since $\tau$ is continuous, strong neighbourhood system $\mathfrak{N}=$ $\left\{\mathcal{N}_{p}(t): t>0, p \in X\right\}$ that determines a Hausdorff and first countable topology for $X$. This topology is called the strong topology for $X$.

Remark 13. Throughout the rest of this paper, we always assume that in a PN space $X$ the triangle function $\tau$ is continuous and $X$ is endowed with strong topology.

Definition 14. A sequence $\left\{p_{n}\right\}_{n \in \mathbb{N}}$ in the PN space $X$ is said to be strongly convergent to a point $p$ in $X$ and one writes $p_{n} \rightarrow p$ or $\lim _{n \rightarrow \infty} p_{n}=p$ if for any $t>0$ there exists a natural number $N$ such that $p_{n} \in \mathcal{N}_{p}(t)$ whenever $n \geq N$.

Definition 15. Given a nonempty set $A$ in the PN space $X$, its probabilistic radius $\mathscr{R}_{A}$ is defined by

$$
\mathscr{R}_{A}(x)= \begin{cases}l^{-1} \phi_{A}(x), & \text { if } x \in[0, \infty), \\ 1, & \text { if } x=\infty,\end{cases}
$$

where $l^{-1} f(x)$ denotes the left limit of the function $f$ at the point $x$ and $\phi_{A}(x)=\inf \left\{N_{p}(x): p \in A\right\}$.

Definition 16. A nonempty set $A$ in a PN space $X$ is said to be

(1) certainly bounded if $\mathscr{R}_{A}\left(x_{0}\right)=1$ for some $x_{0} \in$ $(0, \infty)$

(2) perhaps bounded if $\mathscr{R}_{A}(x)<1$ for every $x \in(0, \infty)$ and $l^{-1} \mathscr{R}_{A}(+\infty)=1$;

(3) perhaps unbounded if $\mathscr{R}_{A}\left(x_{0}\right)>0$ for some $x_{0} \epsilon$ $(0, \infty)$ and $l^{-1} \mathscr{R}_{A}(+\infty) \in(0,1)$;

(4) certainly unbounded if $l^{-1} \mathscr{R}_{A}(+\infty)=0$ that is, if $\mathscr{R}_{A}=\varepsilon_{\infty}$.

Moreover, $A$ is said to be distributionally bounded ( $D$ bounded) if either (1) or (2) holds; that is, if $\mathscr{R}_{A} \in \mathscr{D}^{+}=\{F \in$ $\left.\Delta^{+}: l^{-1} F(+\infty)=1\right\}$; otherwise, if $\mathscr{R}_{A} \in \Delta^{+} \backslash \mathscr{D}^{+}$, then $\mathrm{A}$ is said to be $D$-unbounded.

In the following, we now recall some of the basic concepts related to ideals.

Definition 17. Let $X$ be any nonempty set. A nonempty family $\mathscr{I} \subseteq \mathscr{P}(X)$ is called an ideal in $X$ if

(1) $A, B \in \mathscr{I}$ implies $A \cup B \in \mathscr{I}$;

(2) $A \in \mathscr{I}$ and $B \subseteq A$ imply $B \in \mathscr{I}$.
Definition 18. Let $X$ be any nonempty set. A nonempty family $\mathscr{F} \subseteq \mathscr{P}(X)$ is called a filter in $X$ if

(1) $\emptyset \notin \mathscr{F}$;

(2) $A, B \in \mathscr{F}$ implies $A \cap B \in \mathscr{F}$;

(3) $A \in \mathscr{F}$ and $A \subseteq B$ imply $B \in \mathscr{F}$.

If $\mathscr{I}$ is an ideal in $X$, then $\mathscr{F}(\mathscr{I})=\{X \backslash A: A \in \mathscr{I}\}$ is a filter in $X$, which is called the filter associated with the ideal $\mathscr{I}$. An ideal $\mathscr{I}$ in $X$ is called proper if and only if $X \notin \mathscr{I} . \mathscr{I}$ is called nontrivial if $\mathscr{I} \neq\{\emptyset\}$. An ideal is called an admissible ideal if it is proper and contains $\{x\}$ for all $x \in X$.

Definition 19. An admissible ideal $\mathscr{I}$ is said to satisfy the condition (AP) if, for every countable family of mutually disjoint sets $\left\{A_{1}, A_{2}, \ldots\right\}$ belonging to $\mathscr{F}$, there exists a countable family of sets $\left\{B_{1}, B_{2}, \ldots\right\}$ such that $A_{j} \Delta B_{j}$ is a finite set for every $j \in \mathbb{N}$ and $B=\cup_{j=1}^{\infty} B_{j} \in \mathscr{I}$.

Throughout the paper, $\mathscr{I}$ stands for a nontrivial admissible ideal of $\mathbb{N}$ and $\mathscr{F}(\mathscr{I})$ is the filter associated with the ideal $\mathscr{I}$ of $\mathbb{N}$.

Definition 20 (see [18]). A sequence of real numbers $\left\{x_{n}\right\}_{n \in \mathbb{N}}$ is said to be $\mathscr{I}$-statistically convergent to $x$ if, for each $\epsilon>0$ and $\delta>0$,

$$
\left\{n \in \mathbb{N}: \frac{1}{n}\left|\left\{k \leq n:\left|x_{k}-x\right| \geq \epsilon\right\}\right| \geq \delta\right\} \in \mathscr{I} .
$$

In this case, we write $x_{n} \rightarrow x(S(\mathscr{I}))$.

Definition 21 (see [41]). A sequence $\left\{x_{n}\right\}_{n \in \mathbb{N}}$ in a PM space $(X, \mathfrak{F}, \tau)$ is said to be strong $\mathscr{I}$-statistically convergent to $x$ if, for each $\epsilon>0$ and $\delta>0$,

$$
\left\{n \in \mathbb{N}: \frac{1}{n}\left|\left\{k \leq n: x_{k} \notin \mathscr{N}_{x}(\epsilon)\right\}\right| \geq \delta\right\} \in \mathscr{I} .
$$

In this case, we write $x_{n} \rightarrow x\left(S_{\mathrm{PM}}(\mathscr{I})\right)$ and the class of all strong $\mathscr{I}$-statistically convergent sequences is simply denoted by $S_{\mathrm{PM}}(\mathscr{I})$.

Definition 22 (see [41]). A sequence $\left\{x_{n}\right\}_{n \in \mathbb{N}}$ in a PM space $(X, \mathfrak{F}, \tau)$ is said to be strong $\mathscr{I}$-statistically Cauchy if, for every $\epsilon>0$, there exists a positive integer $N=N(\epsilon)$ such that, for any $\delta>0$,

$$
\left\{n \in \mathbb{N}: \frac{1}{n}\left|\left\{k \leq n: x_{k} \notin \mathscr{N}_{x_{N}}(\epsilon)\right\}\right| \geq \delta\right\} \in \mathscr{I} .
$$

\section{Strong $\mathscr{I}$-Statistical Limit Points and Strong $\mathscr{I}$-Statistical Cluster Points in Probabilistic Normed Spaces}

In this section, we extend the notions of strong statistical limit points and strong statistical cluster points in $\mathrm{PN}$ spaces using ideals. Let $\left(X, \eta, \tau, \tau^{*}\right)$ be a PN space.

Definition 23 (see [36]). Let $\left\{p_{n}\right\}_{n \in \mathbb{N}}$ be a sequence in $X$. We say that a point $p \in X$ is a strong limit point of $\left\{p_{n}\right\}_{n \in \mathbb{N}}$ 
provided that there exists a subsequence of $\left\{p_{n}\right\}_{n \in \mathbb{N}}$ that strongly converges to $p$. We denote the set of all strong limit points of $\left\{p_{n}\right\}_{n \in \mathbb{N}}$ by $L_{s}\left(p_{n}\right)$.

Definition 24. Let $\left\{p_{n}\right\}_{n \in \mathbb{N}}$ be a sequence in $X$ and let $\left\{p_{n_{j}}\right\}_{j \in \mathbb{N}}$ be a subsequence of $\left\{p_{n}\right\}_{n \in \mathbb{N}}$. Denote $K=\left\{n_{1}<n_{2}<\cdots\right\}=$ $\left\{n_{j}: j \in \mathbb{N}\right\}$. If, for all $\delta>0$,

$$
\left\{n \in \mathbb{N}: \frac{1}{n}|\{k \leq n: k \in K\}| \geq \delta\right\} \in \mathscr{I},
$$

then we say that $\left\{p_{n_{j}}\right\}_{j \in \mathbb{N}}$ is an $\mathscr{I}$-statistical thin subsequence of $\left\{p_{n}\right\}_{n \in \mathbb{N}}$. If, for some $\delta>0$,

$$
\left\{n \in \mathbb{N}: \frac{1}{n}|\{k \leq n: k \in K\}| \geq \delta\right\} \notin \mathscr{I}
$$

then $\left\{p_{n_{j}}\right\}_{j \in \mathbb{N}}$ is called an $\mathscr{I}$-statistical nonthin subsequence of $\left\{p_{n}\right\}_{n \in \mathbb{N}}$.

In this sequel, we will abbreviate the subsequence $\left\{p_{n_{k}}\right\}_{k \in \mathbb{N}}$ of $\left\{p_{n}\right\}_{n \in \mathbb{N}}$ as $\{p\}_{K}$, where $K=\left\{n_{1}<n_{2}<\cdots\right\}$.

Definition 25. Let $\left\{p_{n}\right\}_{n \in \mathbb{N}}$ be a sequence in $X$. An element

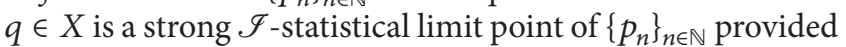
that there exists a set $M=\left\{m_{1}<m_{2}<\cdots\right\} \subset \mathbb{N}$ such that, for some $\delta>0$,

$$
\left\{n \in \mathbb{N}: \frac{1}{n}|\{k \leq n: k \in M\}| \geq \delta\right\} \notin \mathscr{I},
$$

and the subsequence $\left\{p_{m_{k}}\right\}_{k \in \mathbb{N}}$ strongly converges to $q$. We denote the set of all strong $\mathscr{I}$-statistical limit points of $\left\{p_{n}\right\}_{n \in \mathbb{N}}$ by $\Lambda_{S(\mathscr{I})}\left(p_{n}\right)$.

Definition 26. Let $\left\{p_{n}\right\}_{n \in \mathbb{N}}$ be a sequence in $X$. An element $r \in$ $X$ is a strong $\mathscr{I}$-statistical cluster point of $\left\{p_{n}\right\}_{n \in \mathbb{N}}$ provided that for every $\epsilon>0$ there is a $\delta=\delta(\epsilon)>0$ such that

$$
\left\{n \in \mathbb{N}: \frac{1}{n}\left|\left\{k \leq n: d_{L}\left(N_{p_{k}-r}, \varepsilon_{0}\right)<\epsilon\right\}\right| \geq \delta\right\} \notin \mathscr{I} .
$$

We denote the set of all strong $\mathscr{I}$-statistical cluster points of $\left\{p_{n}\right\}_{n \in \mathbb{N}}$ by $\Gamma_{S(\mathscr{F})}\left(p_{n}\right)$.

Theorem 27. For any sequence $\left\{p_{n}\right\}_{n \in \mathbb{N}}$ in $X$, one has $\Lambda_{S(\mathscr{I})}\left(p_{n}\right) \subseteq \Gamma_{S(\mathscr{F})}\left(p_{n}\right) \subseteq L_{s}\left(p_{n}\right)$

Proof. Assume that $q \in \Lambda_{S(\mathscr{F})}\left(p_{n}\right)$. Then, there exists a set $M=\left\{m_{1}<m_{2}<\cdots\right\}$ such that, for some $\delta>0$, say $\delta_{0},\left\{n \in \mathbb{N}:(1 / n)|\{k \leq n: k \in M\}| \geq \delta_{0}\right\} \notin \mathscr{I}$ and the subsequence $\left\{p_{m_{k}}\right\}_{k \in \mathbb{N}}$ of $\left\{p_{n}\right\}_{n \in \mathbb{N}}$ strongly converges to q. Now, for every $\epsilon>0,\left\{n \in \mathbb{N}: d_{L}\left(N_{p_{n}-q}, \varepsilon_{0}\right)<\epsilon\right\} \supseteq$ $M \backslash\left\{m_{k} \in M: d_{L}\left(N_{p_{m_{k}}-q}, \varepsilon_{0}\right) \geq \epsilon\right\}$. Since $p_{m_{k}} \rightarrow q$ strongly, the set $\left\{m_{k} \in M: d_{L}\left(N_{p_{m_{k}}-q}, \varepsilon_{0}\right) \geq \epsilon\right\}$ is finite for every $\epsilon>0$. Let $\epsilon>0$ be given. If possible let, for every $\delta>0$, $\left\{n \in \mathbb{N}:(1 / n)\left|\left\{k \leq n: d_{L}\left(N_{p_{k}-q}, \varepsilon_{0}\right)<\epsilon\right\}\right| \geq \delta\right\} \in \mathscr{J}$. Then, we have

$$
\begin{aligned}
& \left\{n \in \mathbb{N}: \frac{1}{n}\left|\left\{k \leq n: d_{L}\left(N_{p_{k}-q}, \varepsilon_{0}\right)<\epsilon\right\}\right|<\delta\right\} \\
& \subseteq\{n \in \mathbb{N}: \\
& \quad \frac{1}{n}\left|\left\{k \leq n: k \in M \backslash\left\{m_{k} \in \mathbb{N}: d_{L}\left(N_{p_{m_{k}}-q}, \varepsilon_{0}\right) \geq \epsilon\right\}\right\}\right| \\
& \quad<\delta\} \\
& =\left\{n \in \mathbb{N}: \frac{1}{n}|\{k \leq n: k \in M\}|<\delta^{\prime}\right\},
\end{aligned}
$$

where $\delta^{\prime}>\delta+k_{0} / n$ and $k_{0}=\left|\left\{m_{k} \in M: d_{L}\left(N_{p_{m_{k}}-q}, \varepsilon_{0}\right) \geq \epsilon\right\}\right|$. Clearly, $\left\{n \in \mathbb{N}:(1 / n)|\{k \leq n: k \in M\}| \geq \delta^{\prime}\right\} \subseteq\{n \in \mathbb{N}$ : $\left.(1 / n)\left|\left\{k \leq n: d_{L}\left(N_{p_{k}-q}, \varepsilon_{0}\right)<\epsilon\right\}\right| \geq \delta\right\}$. Now, choose $\delta>0$ small enough and $n_{0} \in \mathbb{N}$ in such a way that $\delta+k_{0} / n_{0}<\delta^{\prime}=$ $\delta_{0}$. Then,

$$
\begin{aligned}
& \{n \\
& \left\{\mathbb{N}: \frac{1}{n}|\{k \leq n: k \in M\}| \geq \delta_{0}\right\} \\
& \quad \subset\left\{n \in \mathbb{N}: \frac{1}{n}\left|\left\{k \leq n: d_{L}\left(N_{p_{k}-q}, \varepsilon_{0}\right)<\epsilon\right\}\right| \geq \delta\right\} \\
& \quad \cup\left\{1,2, \ldots, n_{0}\right\} .
\end{aligned}
$$

By our assumption, the set on the right-hand side belongs to $\mathscr{I}$ and so $\left\{n \in \mathbb{N}:(1 / n)|\{k \leq n: k \in M\}| \geq \delta_{0}\right\} \in \mathscr{I}$, which is a contradiction. This implies that there is a $\delta>0$ such that $\left\{n \in \mathbb{N}:(1 / n)\left|\left\{k \leq n: d_{L}\left(N_{p_{k}-q}, \varepsilon_{0}\right)<\epsilon\right\}\right| \geq \delta\right\} \notin \mathscr{I}$. Therefore, $q \in \Gamma_{S(\mathscr{I})}\left(p_{n}\right)$.

Next, let $q \in \Gamma_{S(\mathscr{F})}\left(p_{n}\right)$. Then, for every $\epsilon>0$, there is a $\delta=\delta(\epsilon)>0$ such that $\left\{n \in \mathbb{N}:(1 / n) \mid\left\{k \leq n: d_{L}\left(N_{p_{k}-q}, \varepsilon_{0}\right)<\right.\right.$ $\epsilon\} \mid \geq \delta\} \notin \mathscr{I}$. This means that there are infinitely many terms of the sequence $\left\{p_{n}\right\}_{n \in \mathbb{N}}$ in every strong $\epsilon$-neighbourhood of $q$; that is, $q \in \mathrm{E}_{s}\left(p_{n}\right)$. This completes the proof.

Theorem 28. Let $\left\{p_{n}\right\}_{n \in \mathbb{N}}$ be a sequence in $X$. If $p_{n} \rightarrow$ $p\left(S_{P M}(\mathscr{I})\right)$, then $\Gamma_{S(\mathscr{I})}\left(p_{n}\right)=\{p\}$.

Proof. Let $p_{n} \rightarrow p\left(S_{\mathrm{PM}}(\mathscr{I})\right)$. Then, $\{n \in \mathbb{N}:(1 / n) \mid\{k \leq$ $\left.\left.n: d_{L}\left(N_{p_{k}-p}, \varepsilon_{0}\right) \geq \epsilon\right\} \mid \geq \delta\right\} \in \mathscr{I}$ for all $\epsilon>0$ and $\delta>0$. Clearly, $\left\{n \in \mathbb{N}:(1 / n)\left|\left\{k \leq n: d_{L}\left(N_{p_{k}-p}, \varepsilon_{0}\right)<\epsilon\right\}\right| \geq \delta\right\} \notin \mathscr{I}$. Therefore, $p \in \Gamma_{S(\mathscr{F})}\left(p_{n}\right)$. Now, assume that there exists at least one $r \in \Gamma_{S(\mathscr{J})}\left(p_{n}\right)$ such that $r \neq p$. Since the strong topology is Hausdorff, we can choose $t, t^{\prime}>0$ such that

$$
\left\{n \in \mathbb{N}: d_{L}\left(N_{p_{n}-r}, \varepsilon_{0}\right)<t^{\prime}\right\} \subseteq\left\{n \in \mathbb{N}: d_{L}\left(N_{p_{n}-p}, \varepsilon_{0}\right) \geq t\right\} .
$$

Hence, we get, for any $\delta>0$,

$$
\begin{aligned}
& \left\{n \in \mathbb{N}: \frac{1}{n}\left|\left\{k \leq n: p_{k} \in \mathcal{N}_{r}\left(t^{\prime}\right)\right\}\right| \geq \delta\right\} \\
& \subseteq\left\{n \in \mathbb{N}: \frac{1}{n}\left|\left\{k \leq n: p_{k} \notin \mathcal{N}_{p}(t)\right\}\right| \geq \delta\right\} .
\end{aligned}
$$


Since $p_{n} \rightarrow p\left(S_{\mathrm{PM}}(\mathscr{I})\right)$, the set on the right-hand side belongs to $\mathscr{I}$. This implies that, for the chosen $t^{\prime}>0,\{n \in$ $\left.\mathbb{N}:(1 / n)\left|\left\{k \leq n: d_{L}\left(N_{p_{k}-r}, \varepsilon_{0}\right)<t^{\prime}\right\}\right| \geq \delta\right\} \in \mathscr{I}$ for every $\delta>0$. This contradicts the fact that $r \in \Gamma_{S(\mathscr{I})}\left(p_{n}\right)$. Therefore, we have $\Gamma_{S(\mathscr{I})}\left(p_{n}\right)=\{p\}$.

Theorem 29. For any sequence $\left\{p_{n}\right\}_{n \in \mathbb{N}}$ in $X$, the set $\Gamma_{S(\mathscr{I})}\left(p_{n}\right)$

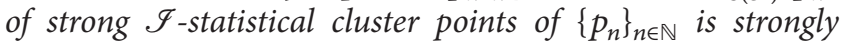
closed.

Proof. Let $p \in \kappa\left(\Gamma_{S(\mathscr{I})}\left(p_{n}\right)\right)$, where $\kappa(A)$ denotes the strong closure of the set $A$ (see [22]). Choose $t>0$. Then, $\Gamma_{S(\mathscr{I})}\left(p_{n}\right) \cap$ $\mathcal{N}_{p}(t) \neq \emptyset$. Let $r \in \Gamma_{S(\mathscr{F})}\left(p_{n}\right) \cap \mathcal{N}_{p}(t)$. Choose $t^{\prime}>0$ in such a way that $\mathcal{N}_{r}\left(t^{\prime}\right) \subseteq \mathcal{N}_{p}(t)$. Since $r \in \Gamma_{S(\mathscr{F})}\left(p_{n}\right)$, there exists a $\delta_{1}>0$ such that $\left\{n \in \mathbb{N}:(1 / n)\left|\left\{k \leq n: p_{k} \in \mathcal{N}_{r}\left(t^{\prime}\right)\right\}\right| \geq \delta_{1}\right\} \notin$ $\mathscr{I}$. Since $\mathcal{N}_{r}\left(t^{\prime}\right) \subseteq \mathcal{N}_{p}(t)$, it follows that

$$
\begin{aligned}
& \left\{n \in \mathbb{N}: \frac{1}{n}\left|\left\{k \leq n: p_{k} \in \mathcal{N}_{r}\left(t^{\prime}\right)\right\}\right| \geq \delta_{1}\right\} \\
& \quad \subseteq\left\{n \in \mathbb{N}: \frac{1}{n}\left|\left\{k \leq n: p_{k} \in \mathcal{N}_{p}(t)\right\}\right| \geq \delta_{1}\right\} .
\end{aligned}
$$

As $\left\{n \in \mathbb{N}:(1 / n)\left|\left\{k \leq n: p_{k} \in \mathcal{N}_{r}\left(t^{\prime}\right)\right\}\right| \geq \delta_{1}\right\} \notin \mathscr{I}$, consequently $\left\{n \in \mathbb{N}:(1 / n)\left|\left\{k \leq n: p_{k} \in \mathcal{N}_{p}(t)\right\}\right| \geq \delta_{1}\right\} \notin$ $\mathscr{I}$. Hence, $p \in \Gamma_{S(\mathscr{J})}\left(p_{n}\right)$; that is, $\kappa\left(\Gamma_{S(\mathscr{I})}\left(p_{n}\right)\right) \subseteq \Gamma_{S(\mathscr{I})}\left(p_{n}\right)$. This proves that $\Gamma_{S(\mathscr{F})}\left(p_{n}\right)$ is strongly closed.

Theorem 30. If $\left\{p_{n}\right\}_{n \in \mathbb{N}}$ and $\left\{q_{n}\right\}_{n \in \mathbb{N}}$ are two sequences in $X$ and there exists a set $M=\left\{m_{1}<m_{2}<\cdots\right\} \subset \mathbb{N}$ such that $\{n \in$ $\mathbb{N}:(1 / n)|\{k \leq n: k \notin M\}| \geq \delta\} \in \mathscr{I}$ and $p_{m_{k}}=q_{m_{k}}$, for all $k \in \mathbb{N}$, then $\Lambda_{S(\mathscr{I})}\left(p_{n}\right)=\Lambda_{S(\mathscr{I})}\left(q_{n}\right)$ and $\Gamma_{S(\mathscr{I})}\left(p_{n}\right)=\Gamma_{S(\mathscr{I})}\left(q_{n}\right)$.

Proof. Assume that $u \in \Lambda_{S(\mathscr{F})}\left(p_{n}\right)$. Let $\{p\}_{K}=\left\{p_{n_{j}}\right\}_{j \in \mathbb{N}}$ be an $\mathscr{I}$-statistical nonthin subsequence of $\left\{p_{n}\right\}$ that strongly converges to $u$, where $K=\left\{n_{1}<n_{2}<\cdots\right\}$. Since $\{n \in \mathbb{N}$ : $(1 / n)|\{k \leq n: k \notin M\}| \geq \delta\} \in \mathcal{I},\{n \in \mathbb{N}:(1 / n) \mid\{k \leq n: k \in$ $K \backslash M\} \mid \geq \delta\} \in \mathscr{I}$. But $(1 / n)|\{k \leq n: k \in K\}|=(1 / n) \mid\{k \leq$ $n: k \in K \cap M\}|+(1 / n)|\{k \leq n: k \in K \backslash M\} \mid$. Hence, $\{n \in \mathbb{N}:(1 / n)|\{k \leq n: k \in K\}| \geq \delta\} \subset\{n \in \mathbb{N}:(1 / n) \mid\{k \leq n:$ $k \in K \cap \mathrm{M}\} \mid \geq \delta / 2\} \cup\{n \in \mathbb{N}:(1 / n)|\{k \leq n: k \in K \backslash M\}| \geq$ $\delta / 2\}$. Since the set on the left-hand side does not belong to $\mathscr{I}$ whereas the second set on the right-hand side belongs to $\mathscr{I}$, the first set on the right-hand side cannot belong to $\mathscr{I}$. This shows that $\{q\}_{K \cap M}$ is a $\mathscr{I}$-statistical nonthin subsequence of $\{q\}_{n \in \mathbb{N}}$ that strongly converges to $u$. Hence, $u \in \Lambda_{S(\mathscr{I})}\left(q_{n}\right)$ and so $\Lambda_{S(\mathscr{F})}\left(p_{n}\right) \subseteq \Lambda_{S(\mathscr{F})}\left(q_{n}\right)$. Similarly, we can prove that $\Lambda_{S(\mathscr{F})}\left(q_{n}\right) \subseteq \Lambda_{S(\mathscr{I})}\left(p_{n}\right)$. Hence, $\Lambda_{S(\mathscr{F})}\left(p_{n}\right)=\Lambda_{S(\mathscr{F})}\left(q_{n}\right)$.

The second assertion $\Gamma_{S(\mathscr{I})}\left(p_{n}\right)=\Gamma_{S(\mathscr{F})}\left(q_{n}\right)$ can be similarly proved.

\section{Strong $\mathscr{I}$-Statistical Continuity in Probabilistic Normed Spaces}

In this section, we introduce the notion of the strong $\mathscr{I}$ statistical continuity and investigate the same for a probabilistic norm, vector addition operation, and scalar multiplication.
Definition 31. Let $\left(X, \eta, \tau, \tau^{*}\right)$ and $\left(Y, \nu, \tau, \tau^{*}\right)$ be two probabilistic normed spaces. A function $f: X \rightarrow Y$ is said to be strongly $\mathscr{I}$-statistically continuous at a point $x_{0} \in X$ if $x_{n} \rightarrow x_{0}\left(S_{\mathrm{PM}}(\mathscr{I})\right)$ implies that $f\left(x_{n}\right) \rightarrow f\left(x_{0}\right)\left(S_{\mathrm{PM}}(\mathscr{I})\right)$.

If $f$ is strongly $\mathscr{I}$-statistically continuous at each point of a set $M \subseteq X$, then $f$ is said to be strongly $\mathscr{I}$-statistically continuous on $M$.

Theorem 32. Let $\left(X, \eta, \tau, \tau^{*}\right)$ be a PN space. Let $X$ be endowed with the strong topology and let $\Delta^{+}$be endowed with the $d_{L^{-}}$ metric topology. Then, $\eta$ is a strongly $\mathscr{I}$-statistically continuous mapping from $X$ to $\Delta^{+}$.

Proof. It is known that the probabilistic norm $\eta$ is a uniformly continuous mapping from $X$ to $\Delta^{+}$; that is, for any $t>0$, there exists a $\lambda>0$ such that $d_{L}\left(N_{p}, N_{p^{\prime}}\right)<t$ whenever $p^{\prime} \in \mathcal{N}_{p}(\lambda)$. Now, let $\left\{p_{n}\right\}_{n \in \mathbb{N}}$ be a sequence in $X$ such that $p_{n} \rightarrow p\left(S_{\mathrm{PM}}(\mathscr{I})\right)$. Then, we have, for each $t>0,\{n \in \mathbb{N}:$ $\left.d_{L}\left(N_{p_{n}}, N_{p}\right) \geq t\right\} \subseteq\left\{n \in \mathbb{N}: p_{n} \notin \mathcal{N}_{p}(\lambda)\right\}$. Clearly, for all $t>0$ and $\delta>0$,

$$
\begin{aligned}
& \left\{n \in \mathbb{N}: \frac{1}{n}\left|\left\{k \leq n: d_{L}\left(N_{p_{k}}, N_{p}\right) \geq t\right\}\right| \geq \delta\right\} \\
& \quad \subseteq\left\{n \in \mathbb{N}: \frac{1}{n}\left|\left\{k \leq n: p_{k} \notin \mathcal{N}_{p}(\lambda)\right\}\right| \geq \delta\right\} .
\end{aligned}
$$

Since $p_{n} \rightarrow p\left(S_{\mathrm{PM}}(\mathscr{I})\right)$, the set on the right-hand side belongs to $\mathscr{I}$. Consequently, for any $t, \delta>0,\{n \in \mathbb{N}$ : $\left.(1 / n)\left|\left\{k \leq n: d_{L}\left(N_{p_{k}}, N_{p}\right) \geq t\right\}\right| \geq \delta\right\} \in \mathscr{I}$. Hence, by definition, we have $N_{p_{n}} \rightarrow N_{p}\left(S_{\mathrm{PM}}(\mathscr{I})\right)$. This means that $\eta$ is a strongly $\mathscr{I}$-statistically continuous mapping.

Theorem 33. Let $\left(X, \eta, \tau, \tau^{*}\right)$ be a PN space. Let $X$ be endowed with the strong topology and let $\Delta^{+}$be endowed with the $d_{L^{-}}$ metric topology. Also assume that $X \times X$ is endowed with the corresponding product topology. Then, vector addition is a strongly $\mathscr{I}$-statistically continuous mapping from $X \times X$ to $X$.

Proof. Let $\left\{p_{n}\right\}_{n \in \mathbb{N}}$ and $\left\{q_{n}\right\}_{n \in \mathbb{N}}$ be two sequences in $X$ such that $p_{n} \rightarrow p\left(S_{\mathrm{PM}}(\mathscr{I})\right)$ and $q_{n} \rightarrow q\left(S_{\mathrm{PM}}(\mathscr{I})\right)$. As $N_{\left(p_{n}+q_{n}\right)-(p+q)} \geq \tau\left(N_{p_{n}-p}, N_{q_{n}-q}\right), d_{L}\left(N_{\left(p_{n}+q_{n}\right)-(p+q)}, \varepsilon_{0}\right) \leq$ $d_{L}\left(\tau\left(N_{p_{n}-p}, N_{q_{n}-q}\right), \varepsilon_{0}\right)$ for every $n \in \mathbb{N}$. Again since continuity of $\tau$ implies its uniform continuity, it follows that for any $t>0$ there is a $\lambda>0$ such that $d_{L}\left(\tau(F, G), \varepsilon_{0}\right)<t$ whenever $d_{L}\left(F, \varepsilon_{0}\right)<\lambda$ and $d_{L}\left(G, \varepsilon_{0}\right)<\lambda$, where $F, G \in$ $\Delta^{+}$. Now, let $t>0$. Then, we can find a $\lambda>0$ such that $d_{L}\left(\tau\left(N_{p_{n}-p}, N_{q_{n}-q}\right), \varepsilon_{0}\right)<t$ whenever $d_{L}\left(N_{p_{n}-p}, \varepsilon_{0}\right)<\lambda$ and $d_{L}\left(N_{q_{n}-q}, \varepsilon_{0}\right)<\lambda$. Hence, $d_{L}\left(N_{\left(p_{n}+q_{n}\right)-(p+q)}, \varepsilon_{0}\right)<t$ whenever $p_{n} \in \mathscr{N}_{p}^{q}(\lambda)$ and $q_{n} \in \mathscr{N}_{q}(\lambda)$. Thus, we have, for all $t>0$,

$$
\begin{aligned}
& \left\{n \in \mathbb{N}: d_{L}\left(N_{\left(p_{n}+q_{n}\right)-(p+q)}, \varepsilon_{0}\right) \geq t\right\} \\
& \quad \subset\left\{n \in \mathbb{N}: p_{n} \notin \mathcal{N}_{p}(\lambda)\right\} \cup\left\{n \in \mathbb{N}: q_{n} \notin \mathcal{N}_{q}(\lambda)\right\} .
\end{aligned}
$$


Therefore, we have, for all $t>0$ and $\delta>0$,

$$
\begin{gathered}
\left\{n \in \mathbb{N}: \frac{1}{n}\left|\left\{k \leq n: d_{L}\left(N_{\left(p_{k}+q_{k}\right)-(p+q)}, \varepsilon_{0}\right) \geq t\right\}\right| \geq \delta\right\} \\
\subset\left\{n \in \mathbb{N}: \frac{1}{n}\left|\left\{k \leq n: p_{k} \notin \mathcal{N}_{p}(\lambda)\right\}\right| \geq \frac{\delta}{2}\right\} \\
\cup\left\{n \in \mathbb{N}: \frac{1}{n}\left|\left\{k \leq n: q_{k} \notin \mathcal{N}_{q}(\lambda)\right\}\right| \geq \frac{\delta}{2}\right\} .
\end{gathered}
$$

Since $p_{n} \rightarrow p\left(S_{\mathrm{PM}}(\mathscr{I})\right)$ and $q_{n} \rightarrow q\left(S_{\mathrm{PM}}(\mathscr{I})\right)$, each set on the right-hand side belongs to $\mathscr{I}$ and so their union also belongs to $\mathscr{I}$. Therefore, we get, for each $t>0$ and $\delta>0,\{n \in$ $\left.\mathbb{N}:(1 / n)\left|\left\{k \leq n: d_{L}\left(N_{\left(p_{k}+q_{k}\right)-(p+q)}, \varepsilon_{0}\right) \geq t\right\}\right| \geq \delta\right\} \in \mathscr{I}$. This shows that $\left(p_{n}+q_{n}\right) \rightarrow(p+q)\left(S_{\mathrm{PM}}(\mathscr{I})\right)$ which completes the proof.

Corollary 34. Let $\left(X, \eta, \tau, \tau^{*}\right)$ be a PN space. The mapping $v$ from $X \times X$ to $\Delta^{+}$defined as $\nu(p, q)=N_{p+q}$ for any $p, q \in X$ is strongly $\mathscr{I}$-statistically continuous.

Proof. Proof of this result immediately follows from Theorems 32 and 33 .

We now investigate the strong $\mathscr{I}$-statistical continuity properties of scalar multiplication given by $\mathscr{M}(\alpha, p)=\alpha p$ for all $\alpha \in \mathbb{R}$ and $p \in X$.

Lemma 35 (see [37]). For any $\alpha \in \mathbb{R}, r \in X$, and $h>0$, there exists a $\lambda>0$ such that $d_{L}\left(N_{\alpha r}, \varepsilon_{0}\right)<h$ whenever $d_{L}\left(N_{r}, \varepsilon_{0}\right)<$ $\lambda$.

Theorem 36. The mapping $\mathscr{M}$ is strongly $\mathscr{I}$-statistically continuous in its second place; that is, for a fixed $\alpha \in \mathbb{R}$, scalar multiplication is a strongly $\mathscr{I}$-statistically continuous mapping from $X$ to $X$.

Proof. Let $\alpha \in \mathbb{R}$ be fixed and let $\left\{p_{n}\right\}_{n \in \mathbb{N}}$ be a sequence in $X$ such that $p_{n} \rightarrow p\left(S_{\mathrm{PM}}(\mathscr{I})\right)$. Then, by Lemma 35, for any $h>0$, we can find a $\lambda>0$ such that $\left\{n \in \mathbb{N}: d_{L}\left(N_{p_{n}-p}, \varepsilon_{0}\right)<\right.$ $\lambda\} \subseteq\left\{n \in \mathbb{N}: d_{L}\left(N_{\alpha\left(p_{n}-p\right)}, \varepsilon_{0}\right)<h\right\}$. Therefore, for any $\delta>0$,

$$
\begin{aligned}
& \left\{n \in \mathbb{N}: \frac{1}{n}\left|\left\{k \leq n: d_{L}\left(N_{\alpha\left(p_{k}-p\right)}, \varepsilon_{0}\right) \geq h\right\}\right| \geq \delta\right\} \\
& \subseteq\left\{n \in \mathbb{N}: \frac{1}{n}\left|\left\{k \leq n: d_{L}\left(N_{p_{k}-p}, \varepsilon_{0}\right) \geq \lambda\right\}\right| \geq \delta\right\} .
\end{aligned}
$$

Since $p_{n} \rightarrow p\left(S_{\mathrm{PM}}(\mathscr{I})\right)$, the set on the right-hand side belongs to $\mathscr{I}$. Therefore, for any $h>0$ and $\delta>0,\{n \in \mathbb{N}$ : $\left.(1 / n)\left|\left\{k \leq n: d_{L}\left(N_{\alpha}\left(p_{k}-p\right), \varepsilon_{0}\right) \geq h\right\}\right| \geq \delta\right\} \in \mathscr{I}$. Hence, $\alpha p_{n} \rightarrow \alpha p\left(S_{\mathrm{PM}}(\mathscr{I})\right)$.

However, in general, the mapping $\mathscr{M}$ needs not to be strongly $\mathscr{I}$-statistically continuous in its first place.
Example 37. Let $X$ be the real line $\mathbb{R}$ viewed as a onedimensional linear space and let $\tau=\tau_{W}$ and $\tau^{*}=\tau_{M}$, where $\tau_{W}$ and $\tau_{M}$ are the continuous triangle functions defined by

$$
\begin{gathered}
\left(\tau_{W}(F, G)\right)(t)=\sup \{\max \{F(u)+G(v)-1,0\}: u+v=t\}, \\
\left(\tau_{M}(F, G)\right)(t)=\sup \{\min \{F(u), G(v)\}: u+v=t\} .
\end{gathered}
$$

For $p \in \mathbb{R}$, define $\eta$ by setting $\eta(0)=\varepsilon_{0}$ and

$$
\eta(p)=N_{p}=\frac{|p|+1}{|p|+2} \varepsilon_{0}+\frac{1}{|p|+2} \varepsilon_{\infty}, \quad \text { for } p \neq 0 .
$$

Clearly, $\left(X, \eta, \tau, \tau^{*}\right)$ is a PN space. Choose an infinite set $A \epsilon$ $\mathscr{I}$. Now, consider the real sequence $\left\{\alpha_{n}\right\}_{n \in \mathbb{N}}$ defined by

$$
\alpha_{n}= \begin{cases}1, & \text { if } n \in A \\ \frac{1}{n}, & \text { if } n \in \mathbb{N} \backslash A\end{cases}
$$

It can be easily shown that $\alpha_{n} \rightarrow 0\left(S_{\mathrm{PM}}(\mathscr{I})\right)$ but $d_{L}\left(N_{\alpha_{n} p}, \varepsilon_{0}\right) \nrightarrow 0\left(S_{\mathrm{PM}}(\mathscr{I})\right)$. This example shows that the mapping from $\mathbb{R}$ into $X$ defined by $\alpha \mapsto \alpha p$ is not strongly $\mathscr{I}$-statistically continuous for any fixed $p \in X$; that is, the mapping $\mathscr{M}$ is not strongly $\mathscr{I}$-statistically continuous in its first place.

A triangle function $\tau^{*}$ is called Archimedean if $\tau^{*}$ admits no idempotents other than $\epsilon_{0}$ and $\epsilon_{\infty}$. More details on Archimedean triangle function can be found in the book [22]. If $\tau^{*}$ is Archimedean, then we can establish the following lemmas.

Lemma 38 (see [37]). If $\tau^{*}$ is Archimedean, then, for any $p \in$ $X$ such that $N_{p} \neq \varepsilon_{\infty}$ and any $h>0$, there exists $a>0$ such that $d_{L}\left(N_{\alpha p}, \varepsilon_{0}\right)<h$ whenever $|\alpha|<\beta$.

Theorem 39. If $\left(X, \eta, \tau, \tau^{*}\right)$ is PN space such that $\tau^{*}$ is Archimedean and if $N_{p} \neq \varepsilon_{\infty}$ for every $p \in X$, then for any fixed $p \in X$ the mapping $\mathscr{M}$ is strongly $\mathscr{I}$-statistically continuous in its first place.

Proof. Let $p \in X$ be fixed and let $\left\{\alpha_{n}\right\}_{n \in \mathbb{N}}$ be a real sequence such that $\alpha_{n} \rightarrow \alpha(S(\mathscr{I}))$. Let $h>0$ be given. By Lemma 38, we can find a $\beta>0$ such that $d_{L}\left(N_{(r-\alpha) p}, \varepsilon_{0}\right)<h$ whenever $|r-\alpha|<\beta$. In particular, $\left|\alpha_{n}-\alpha\right|<\beta$ implies that $d_{L}\left(N_{\left(\alpha_{n}-\alpha\right) p}, \varepsilon_{0}\right)<h$. Therefore, $\left\{n \in \mathbb{N}: d_{L}\left(N_{\left(\alpha_{n}-\alpha\right) p}, \varepsilon_{0}\right) \geq\right.$ $h\} \subseteq\left\{n \in \mathbb{N}:\left|\alpha_{n}-\alpha\right| \geq \beta\right\}$. It now readily follows that, for all $\delta>0$,

$$
\begin{gathered}
\left\{n \in \mathbb{N}: \frac{1}{n}\left|\left\{k \leq n: d_{L}\left(N_{\left(\alpha_{k}-\alpha\right) p}, \varepsilon_{0}\right) \geq h\right\}\right| \geq \delta\right\} \\
\subseteq\left\{n \in \mathbb{N}: \frac{1}{n}\left|\left\{k \leq n:\left|\alpha_{k}-\alpha\right| \geq \beta\right\}\right| \geq \delta\right\} .
\end{gathered}
$$

Since $\alpha_{n} \rightarrow \alpha(S(\mathscr{I}))$, the set on the right-hand side belongs to $\mathscr{I}$ and, consequently, for any $\delta>0$ and $h>0,\{n \in \mathbb{N}$ : $\left.(1 / n)\left|\left\{k \leq n: d_{L}\left(N_{\left(\alpha_{k}-\alpha\right) p}, \varepsilon_{0}\right) \geq h\right\}\right| \geq \delta\right\} \in \mathscr{I}$. Therefore, $\alpha_{n} p \rightarrow \alpha p\left(S_{\mathrm{PM}}(\mathscr{I})\right)$ as desired. 

result.

The following lemmas will be needed to prove our next

Lemma 40 (see [37]). If $0 \leq \alpha \leq \beta$, then $N_{\beta p} \leq N_{\alpha p}$ for any $p \in X$.

Lemma 41 (see [37]). Let $\tau$ be a continuous triangle function and let $S$ be the set of all triplets $(F, G, H)$ in $\Delta^{+} \times \Delta^{+} \times \Delta^{+}$such that $F \geq \tau(H, G)$ and $G \geq \tau(H, F)$. Then, for any $h>0$, there exists $a \lambda>0$ such that if $(F, G, H)$ is in $S$ and $d_{L}\left(H, \varepsilon_{0}\right)<\lambda$, then $d_{L}(F, G)<h$.

Theorem 42. Let $\left(X, \eta, \tau, \tau^{*}\right)$ be a PN space such that $\tau^{*}$ is Archimedean and $N_{p} \neq \varepsilon_{\infty}$ for all $p \in X$. Then, the scalar multiplication is a jointly strong $\mathscr{I}$-statistically continuous mapping from $\mathbb{R} \times X$ endowed with the natural product topology onto $X$. Furthermore, the mapping $\mu^{\prime}: \mathbb{R} \times X \rightarrow \Delta^{+}$ given by $\mu^{\prime}(\alpha, p)=\eta(\alpha p)=N_{\alpha p}$ for any $\alpha \in \mathbb{R}$ and any $p \in X$ is also jointly strong $\mathscr{I}$-statistically continuous.

Proof. Let $\left\{p_{n}\right\}_{n \in \mathbb{N}}$ be a sequence in $X$ such that $p_{n} \rightarrow$ $p\left(S_{\mathrm{PM}}(\mathscr{F})\right)$ and let $\left\{\alpha_{n}\right\}_{n \in \mathbb{N}}$ be a real sequence such that $\alpha_{n} \rightarrow \alpha(S(\mathscr{I}))$. Consider the set $M_{1}=\left\{n \in \mathbb{N}:\left|\alpha_{n}-\alpha\right|<1\right\}$. Since $\alpha_{n} \rightarrow \alpha(S(\mathscr{I}))$, we have, for every $\delta>0$, $\left\{n \in \mathbb{N}:(1 / n)\left|\left\{k \leq n:\left|\alpha_{k}-\alpha\right| \geq 1\right\}\right| \geq \delta\right\} \in \mathscr{F}$; that is, $\left\{n \in \mathbb{N}:(1 / n)\left|\left\{k \leq n: k \notin M_{1}\right\}\right| \geq \delta\right\} \in \mathscr{I}$. Now, if $n \in M_{1}$, then $\left|\alpha_{n}\right|<|\alpha|+1$. From the properties of probabilistic norm, we have $N_{\left(\alpha_{n} p_{n}-\alpha p\right)} \geq \tau\left(N_{\alpha_{n}\left(p_{n}-p\right)}, N_{\left(\alpha_{n}-\alpha\right) p}\right)=$ $\tau\left(N_{\left|\alpha_{n}\right|\left(p_{n}-p\right)}, N_{\left(\alpha_{n}-\alpha\right) p}\right) \geq \tau\left(N_{(|\alpha|+1)\left(p_{n}-p\right)}, N_{\left(\alpha_{n}-\alpha\right) p}\right)$. Thus, if $n \in M_{1}$, then we have $d_{L}\left(N_{\left(\alpha_{n} p_{n}-\alpha p\right)}, \varepsilon_{0}\right) \leq$ $d_{L}\left(\tau\left(N_{(|\alpha|+1)\left(p_{n}-p\right)}, N_{\left(\alpha_{n}-\alpha\right) p}\right), \varepsilon_{0}\right)$. Next, let $t \stackrel{>}{>} 0$. Since $\tau$ is uniformly continuous, we can find a $\lambda>0$ such that $d_{L}\left(\tau\left(N_{(|\alpha|+1)\left(p_{n}-p\right)}, N_{\left(\alpha_{n}-\alpha\right) p}\right), \varepsilon_{0}\right)<t$ whenever $d_{L}\left(N_{(|\alpha|+1)\left(p_{n}-p\right)}, \varepsilon_{0}\right)<\lambda$ and $d_{L}\left(N_{\left(\alpha_{n}-\alpha\right) p}, \varepsilon_{0}\right)<\lambda$. For such a $\lambda>0$, consider the sets

$$
\begin{aligned}
& M_{2}=\left\{n \in \mathbb{N}: d_{L}\left(N_{(|\alpha|+1)\left(p_{n}-p\right)}, \varepsilon_{0}\right)<\lambda\right\}, \\
& M_{3}=\left\{n \in \mathbb{N}: d_{L}\left(N_{\left(\alpha_{n}-\alpha\right) p}, \varepsilon_{0}\right)<\lambda\right\} .
\end{aligned}
$$

If $k \in M_{1} \cap M_{2} \cap M_{3}$, then clearly $d_{L}\left(N_{\left(\alpha_{k} p_{k}-\alpha p\right)}, \varepsilon_{0}\right)<t$. Hence, $\left\{n \in \mathbb{N}: d_{L}\left(N_{\left(\alpha_{n} p_{n}-\alpha p\right)}, \epsilon_{0}\right) \geq t\right\} \subseteq\{n \in \mathbb{N}$ : $\left.d_{L}\left(N_{(|\alpha|+1)\left(p_{n}-p\right)}, \varepsilon_{0}\right) \geq \lambda\right\} \cup\left\{n \in \mathbb{N}: d_{L}\left(N_{\left(\alpha_{n}-\alpha\right) p}, \varepsilon_{0}\right) \geq\right.$ $\lambda\} \cup\left\{n \in \mathbb{N}:\left|\alpha_{n}-\alpha\right| \geq 1\right\}$. Consequently, we can write $(1 / n)\left|\left\{k \leq n: d_{L}\left(N_{\left(\alpha_{k} p_{k}-\alpha p\right)}, \varepsilon_{0}\right) \geq t\right\}\right| \leq(1 / n) \mid\{k \leq n:$ $\left.d_{L}\left(N_{(|\alpha|+1)\left(p_{k}-p\right)}, \varepsilon_{0}\right) \geq \lambda\right\}|+(1 / n)|\left\{k \leq n: d_{L}\left(N_{\left(\alpha_{k}-\alpha\right) p}, \varepsilon_{0}\right) \geq\right.$ $\lambda\}|+(1 / n)|\left\{k \leq n:\left|\alpha_{k}-\alpha\right| \geq 1\right\} \mid$. Then, for any $\delta>0$,

$$
\begin{aligned}
& \left\{n \in \mathbb{N}: \frac{1}{n}\left|\left\{k \leq n: d_{L}\left(N_{\left(\alpha_{k} p_{k}-\alpha p\right)}, \varepsilon_{0}\right) \geq t\right\}\right| \geq \delta\right\} \\
& \subset\{n \in \mathbb{N}: \\
& \quad \frac{1}{n}\left|\left\{k \leq n: d_{L}\left(\tau\left(N_{(|\alpha|+1)\left(p_{k}-p\right)}, N_{\left(\alpha_{k}-\alpha\right) p}\right), \varepsilon_{0}\right) \geq t\right\}\right| \\
& \quad \geq \delta\} \\
& \subseteq\left\{n \in \mathbb{N}: \frac{1}{n}\left|\left\{k \leq n: d_{L}\left(N_{(|\alpha|+1)\left(p_{k}-p\right)}, \varepsilon_{0}\right) \geq \lambda\right\}\right| \geq \frac{\delta}{3}\right\}
\end{aligned}
$$

$$
\begin{aligned}
& \cup\left\{n \in \mathbb{N}: \frac{1}{n}\left|\left\{k \leq n: d_{L}\left(N_{\left(\alpha_{k}-\alpha\right) p}, \varepsilon_{0}\right) \geq \lambda\right\}\right| \geq \frac{\delta}{3}\right\} \\
& \cup\left\{n \in \mathbb{N}: \frac{1}{n}\left|\left\{k \leq n:\left|\alpha_{k}-\alpha\right| \geq 1\right\}\right| \geq \frac{\delta}{3}\right\} .
\end{aligned}
$$

Clearly, all the three sets on the right-hand side of the expression belong to $\mathscr{I}$. Therefore, for any $\delta>0,\{n \in \mathbb{N}$ : $\left.(1 / n)\left|\left\{k \leq n: d_{L}\left(N_{\left(\alpha_{k} p_{k}-\alpha p\right)}, \varepsilon_{0}\right) \geq t\right\}\right| \geq \delta\right\} \in \mathscr{I}$ which shows that $\alpha_{n} p_{n} \rightarrow \alpha p\left(S_{\mathrm{PM}}(\mathscr{I})\right)$. This completes the proof of the first part of the theorem.

Let us now show that the mapping $\mu^{\prime}$ is jointly strong $\mathscr{I}$-statistically continuous. Assume that $p_{n} \rightarrow p\left(S_{\mathrm{PM}}(\mathscr{I})\right)$ and $\alpha_{n} \rightarrow \alpha(S(\mathscr{I}))$. Then, we have $\alpha_{n} p_{n} \rightarrow \alpha p\left(S_{\mathrm{PM}}(\mathscr{I})\right)$. Therefore, $d_{L}\left(N_{\alpha_{n} p_{n}-\alpha p}, \varepsilon_{0}\right) \rightarrow 0(S(\mathscr{I}))$. Now, we can write, for every $n \in \mathbb{N}, N_{\alpha_{n} p_{n}} \geq \tau\left(N_{\alpha_{n} p_{n}-\alpha p}, N_{\alpha p}\right)$ and $N_{\alpha p} \geq$ $\tau\left(N_{\alpha p-\alpha_{n} p_{n}}, N_{\alpha_{n} p_{n}}\right)$. By Lemma 41, we can say that for any $h>$ 0 there exists a $\lambda>0$ such that $d_{L}\left(N_{\alpha_{n} p_{n}}, N_{\alpha p}\right)<h$ whenever $d_{L}\left(N_{\alpha_{n} p_{n}-\alpha p}, \varepsilon_{0}\right)<\lambda$. Now, using the argument similar to that of the preceding proof, we obtain $d_{L}\left(N_{\alpha_{n} p_{n}}, N_{\alpha p}\right) \rightarrow$ $0(S(\mathscr{F}))$. Hence, it follows that $\mu^{\prime}$ is jointly strong $\mathscr{I}$ statistically continuous.

\section{Strong $\mathscr{I}$-Statistically $D$-Bounded Sequences in Probabilistic Normed Spaces}

Definition 43 (see [38]). Let $\left(X, \eta, \tau, \tau^{*}\right)$ be a PN space. A sequence $\left\{p_{n}\right\}_{n \in \mathbb{N}}$ in $X$ is statistically $D$-bounded provided that there exists a set $K=\left\{n_{1}<n_{2}<\cdots\right\} \subset \mathbb{N}$ with $\delta(K)=\lim _{n \rightarrow \infty}(1 / n)|\{k \leq n: k \in K\}|=1$ such that $\left\{p_{n_{k}}\right\}_{k \in \mathbb{N}}$ is $D$-bounded.

In this section, we generalize the above definition for sequences in a PN space and introduce the concept of a strongly $\mathscr{I}$-statistically $D$-bounded sequence.

Definition 44. Let $\left(X, \eta, \tau, \tau^{*}\right)$ be a PN space. A sequence $\left\{p_{n}\right\}_{n \in \mathbb{N}}$ in $X$ is strongly $\mathscr{I}$-statistically $D$-bounded provided that there exists a set $K=\left\{n_{1}<n_{2}<\cdots\right\} \subset \mathbb{N}$ such that $\{n \in \mathbb{N}:(1 / n)|\{k \leq n: k \notin K\}| \geq \delta\} \in \mathscr{I}$ for any $\delta>0$ and $\left\{p_{n_{k}}\right\}_{k \in \mathbb{N}}$ is $D$-bounded.

Clearly, in this case, $\mathscr{R}_{\left\{p_{n_{k}}: k \in \mathbb{N}\right\}}=\mathscr{R}_{\{\}_{K}} \in \mathscr{D}^{+}$. Note that a $D$-bounded sequence is always strongly $\mathscr{I}$-statistically $D$ bounded, but the converse is not generally true.

Theorem 45. A sequence $\left\{p_{n}\right\}_{n \in \mathbb{N}}$ in the $P N$ space $X$ is strongly $\mathscr{I}$-statistically $D$-bounded if and only if there exists a set $K=$ $\left\{n_{1}<n_{2}<\cdots\right\}$ with $\{n \in \mathbb{N}:(1 / n)|\{k \leq n: k \notin K\}| \geq \delta\} \in \mathscr{I}$ for any $\delta>0$ and a distribution function $G \in \mathscr{D}^{+}$such that $N_{p_{n_{k}}} \geq G$.

Proof. The proof of the theorem immediately follows from Theorem 2.1 of [29] and Definition 44.

Example 46. Let us consider the simple space $(\mathbb{R},|\cdot|, G, M)$, where $|\cdot|$ denotes the usual norm on $\mathbb{R} ; G \in \Delta^{+}, G \neq \varepsilon_{0}, \varepsilon_{\infty}$, 
and the probabilistic norm $\eta: \mathbb{R} \rightarrow \Delta^{+}$is given by $\eta(0)=\varepsilon_{0}$ and, for $t>0, p \neq 0$,

$$
\eta_{p}(t)=N_{p}(t)=G\left(\frac{t}{|p|}\right) .
$$

This space is called Menger PN space under $M$ where $M$ is the $t$-norm defined by $M(x, y)=\min \{x, y\}$. Now, assume that there is a $x_{0} \in(0, \infty)$ such that $G\left(x_{0}\right)=1$ and $l^{+} G(0)=0$.

Next, assume that $\mathbb{N}=\cup_{j=1}^{\infty} D_{j}$ is a decomposition of $\mathbb{N}$ (i.e., $D_{k} \cap D_{l}=\emptyset$ for $\left.k \neq l\right)$ where $D_{j}(j=1,2,3, \ldots)$ are infinite sets defined as $D_{j}=\left\{2^{j-1}(2 s-1): s \in \mathbb{N}\right\}$. Denote by $\mathscr{I}$ the class of all $A \subset \mathbb{N}$ such that $A$ intersects only a finite number of $D_{j}^{\prime} s$. It can be easily verified that $\mathscr{I}$ is an admissible ideal. Note that $\left|D_{j}(1, n)\right| / n=(1 / n)\left|\left\{k \leq n: k \in D_{j}\right\}\right| \leq$ $1 / 2^{j-1}$ for all $j \in \mathbb{N}$. Now, for every $\delta>0$, there exists a $j_{0} \in \mathbb{N}$ such that $\left|D_{j_{0}}(1, n)\right| / n<1 / 2^{j_{0}-1}<\delta$. Therefore, $\{n \in \mathbb{N}$ : $\left.\left|D_{j_{0}}(1, n)\right| / n \geq \delta\right\} \subseteq\left\{D_{1} \cup D_{2} \cup \cdots \cup D_{j_{0}-1}\right\}=A$ (say). Clearly, $A \in \mathscr{I}$. We define a sequence $\left\{p_{n}\right\}_{n \in \mathbb{N}}$ in the $\mathrm{PN}$ space $(\mathbb{R},|\cdot|$, $G, M)$ by

$$
p_{n}= \begin{cases}n & \text { if } n \in D_{j_{0}} \\ 1 & \text { if } n \notin D_{j_{0}}\end{cases}
$$

The subsequence $\{p\}_{D_{j_{0}}^{c}}$, where $c$ stands for the complement, is certainly bounded and hence $D$-bounded in $(\mathbb{R},|\cdot|, G, M)$. Moreover, the subsequence $\{p\}_{D_{j_{0}}}$ is certainly unbounded in $(\mathbb{R},|\cdot|, G, M)$. Therefore, the sequence $\left\{p_{n}\right\}_{n \in \mathbb{N}}$ is strongly $\mathscr{I}$ statistically $D$-bounded, but it is not statistically $D$-bounded as $\delta\left(D_{j_{0}}\right) \neq 0$.

We now present certain results which are modifications of similar results proved for statistically $D$-bounded sequences [38]. The proofs of these results are parallel to the corresponding results of [38] with necessary modifications.

Theorem 47. If $\left\{p_{n}\right\}_{n \in \mathbb{N}}$ is a strongly $\mathscr{I}$-statistically $D$ bounded sequence in the PN space $\left(X, \eta, \tau, \tau^{*}\right)$, then there exists a D-bounded sequence $\left\{q_{n}\right\}_{n \in \mathbb{N}}$ and a strongly $\mathscr{I}_{\text {-statistically }}$ null sequence $\left\{r_{n}\right\}_{n \in \mathbb{N}}$ such that $p_{n}=q_{n}+r_{n}$ for all $n \in \mathbb{N}$.

Proof. Let $\left\{p_{n}\right\}_{n \in \mathbb{N}}$ be a strongly $\mathscr{I}$-statistically $D$-bounded sequence in the PN space. Then, there exists a set $K=\left\{n_{1}<\right.$ $\left.n_{2}<\cdots\right\} \subset \mathbb{N}$ with $\{n \in \mathbb{N}:(1 / n)|\{k \leq n: k \notin K\}| \geq \delta\} \in \mathscr{I}$, for any $\delta>0$, such that $\{p\}_{K}$ is $D$-bounded. Now, define

$$
\begin{aligned}
& q_{n}= \begin{cases}p_{n}, & \text { if } n \in K, \\
\theta, & \text { otherwise, }\end{cases} \\
& r_{n}= \begin{cases}\theta, & \text { if } n \in K, \\
p_{n}, & \text { otherwise }\end{cases}
\end{aligned}
$$

We have, for each $\epsilon>0$ and $\delta>0$,

$$
\begin{aligned}
& \left\{n \in \mathbb{N}: \frac{1}{n}\left|\left\{k \leq n: d_{L}\left(N_{r_{k}-\theta}, \varepsilon_{0}\right) \geq \epsilon\right\}\right| \geq \delta\right\} \\
& \subseteq\left\{n \in \mathbb{N}: \frac{1}{n}|\{k \leq n: k \notin K\}| \geq \delta\right\} .
\end{aligned}
$$

Since the set on the right-hand side belongs to $\mathscr{I},\{n \in \mathbb{N}$ : $\left.(1 / n)\left|\left\{k \leq n: d_{L}\left(N_{r_{k}-\theta}, \varepsilon_{0}\right) \geq \epsilon\right\}\right| \geq \delta\right\} \in \mathscr{I}$. Thus, $r_{k} \rightarrow$ $\theta\left(S_{\mathrm{PM}}(\mathscr{I})\right)$. The sequence $\left\{q_{n}\right\}_{n \in \mathbb{N}}$ is clearly $D$-bounded. It is easy to see that $p_{n}=q_{n}+r_{n}$, where $\left\{q_{n}\right\}_{n \in \mathbb{N}}$ is $D$-bounded and $r_{n} \rightarrow \theta\left(S_{\mathrm{PM}}(\mathscr{I})\right)$.

Theorem 48. Let $\left(X, \eta, \tau, \tau^{*}\right)$ be a PN space in which $\eta(X) \subseteq$ $\mathscr{D}^{+}$and $\mathscr{D}^{+}$is invariant under $\tau$; that is, $\tau\left(\mathscr{D}^{+} \times \mathscr{D}^{+}\right) \subseteq \mathscr{D}^{+}$. If $\left\{p_{n}\right\}_{n \in \mathbb{N}}$ is a sequence in $X$ such that $p_{n} \rightarrow p\left(S_{P M}(\mathscr{I})\right)$, then $\left\{p_{n}\right\}_{n \in \mathbb{N}}$ is strongly $\mathscr{I}$-statistically D-bounded.

Proof. Let $p_{n} \rightarrow p\left(S_{\mathrm{PM}}(\mathscr{I})\right)$. Then, for each $G \in \mathscr{D}^{+} \backslash\left\{\varepsilon_{0}\right\}$, there exists a set $K=\left\{n_{1}<n_{2}<\cdots\right\}$ with $\{n \in \mathbb{N}:(1 / n) \mid\{k \leq$ $n: k \notin K\} \mid \geq \delta\} \in \mathscr{I}$, for any $\delta>0$, such that $N_{p_{n_{k}}-p} \geq G$. Therefore, $N_{p_{n_{k}}} \geq \tau\left(N_{p_{n_{k}}-p}, N_{p}\right) \geq \tau\left(G, N_{p}\right)$ for all $n_{k} \in K$. By our assumption, we have $\tau\left(G, N_{p}\right) \in \mathscr{D}^{+}$and now the result follows from Theorem 45 .

Theorem 49. Let $\left(X, \eta, \tau, \tau^{*}\right)$ be a PN space in which $\eta(X) \subseteq$ $\mathscr{D}^{+}$and $\mathscr{D}^{+}$is invariant under $\tau$. If $\left\{p_{n}\right\}_{n \in \mathbb{N}}$ is strongly $\mathscr{I}$ statistically Cauchy sequence in $X$, then it is strongly $\mathscr{I}$ statistically D-bounded.

Proof. Let $\left\{p_{n}\right\}_{n \in \mathbb{N}}$ be strongly $\mathscr{I}$-statistically Cauchy. Then, for every $G \in \mathscr{D}^{+} \backslash\left\{\varepsilon_{0}\right\}$, there exists a set $K=\left\{n_{1}<n_{2}<\cdots\right\}$ for which $\{n \in \mathbb{N}:(1 / n)|\{k \leq n: k \notin K\}| \geq \delta\} \in \mathscr{I}$ for any $\delta>0$ and a $N \in \mathbb{N}$, such that $N_{p_{n_{k}}-p_{N}} \geq G$. Let $G \in \mathscr{D}^{+} \backslash\left\{\varepsilon_{0}\right\}$ be given, and then we can find a $N \in \mathbb{N}$ such that $N_{p_{n_{k}}} \geq$ $\tau\left(N_{p_{n_{k}}-p_{N}}, N_{p_{N}}\right) \geq \tau\left(G, N_{p_{N}}\right)$. By our assumption, we have $\tau\left(G, N_{p_{n}}\right) \in \mathscr{D}^{+}$and the rest follows from Theorem 45 .

Theorem 50. Let $\left\{p_{n}\right\}_{n \in \mathbb{N}}$ be a strongly $\mathscr{I}$-statistically $D$ bounded sequence in the PN space $\left(X, \eta, \tau, \tau^{*}\right)$. If $\mathscr{D}^{+}$is invariant under $\tau$, then the sequence $\left\{\alpha p_{n}\right\}_{n \in \mathbb{N}}$ is strongly $\mathscr{I}$ statistically $D$-bounded for every fixed $\alpha \in \mathbb{R}$.

Proof. By axiom (N2), it is sufficient to consider the case $\alpha \geq$ 0 . If $\alpha=0$ or $\alpha=1$, then $\left\{\alpha p_{n}\right\}_{n \in \mathbb{N}}$ is strongly $\mathscr{I}$-statistically $D$-bounded. Let $\alpha \in(0,1)$. Since $\left\{p_{n}\right\}_{n \in \mathbb{N}}$ is strongly $\mathscr{I}$ statistically $D$-bounded, there exists a set $K=\left\{n_{1}<n_{2}<\right.$ $\cdots\} \subset \mathbb{N}$ such that $\{n \in \mathbb{N}:(1 / n)|\{k \leq n: k \notin K\}| \geq \delta\} \in \mathscr{I}$ for any $\delta>0$ and $\mathscr{R}_{\{p\}_{K}} \in \mathscr{D}^{+}$. Thus, we have $N_{\alpha p_{n_{k}}} \geq$ $N_{p_{n_{k}}} \geq \mathscr{R}_{\{p\}_{K}}$ which means that $\mathscr{R}_{\{\alpha p\}_{K}} \in \mathscr{D}^{+}$, where $\{\alpha p\}_{K}=$ $\left\{\alpha p_{n_{k}}\right\}_{k \in \mathbb{N}}$. Therefore, $\left\{\alpha p_{n}\right\}_{n \in \mathbb{N}}$ is strongly $\mathscr{I}$-statistically $D$ bounded. Now, let $\alpha>1$ and set $m=\lfloor|\alpha|\rfloor+1$. Then, $N_{\alpha p_{n_{k}}} \geq$ $N_{m p_{n_{k}}} \geq \tau\left(N_{(m-1) p_{n_{k}}}, N_{p_{n_{k}}}\right) \geq \tau\left(\tau\left(N_{(m-2) p_{n_{k}}}, N_{p_{n_{k}}}\right), N_{p_{n_{k}}}\right) \geq$ $\tau^{m-1}\left(N_{p_{n_{k}}}, \ldots, N_{p_{n_{k}}}\right) \geq \tau^{m-1}\left(\mathscr{R}_{\{p\}_{K}}, \ldots, \mathscr{R}_{\{p\}_{K}}\right)$, where $\tau^{m-1}\left(\mathscr{R}_{\{p\}_{K}}, \ldots, \mathscr{R}_{\{p\}_{K}}\right) \in \mathscr{D}^{+}$. Hence, $\mathscr{R}_{\{\alpha p\}_{K}} \in \mathscr{D}^{+}$which completes the proof.

Theorem 51. Let $\left\{p_{n}\right\}_{n \in \mathbb{N}}$ and $\left\{q_{n}\right\}_{n \in \mathbb{N}}$ be two strongly $\mathscr{I}$ statistically D-bounded sequences in the PN space $\left(X, \eta, \tau, \tau^{*}\right)$. If $\mathscr{D}^{+}$is invariant under $\tau$, then the sequence $\left\{p_{n}+q_{n}\right\}_{n \in \mathbb{N}}$ is also strongly $\mathscr{I}$-statistically $D$-bounded.

Proof. Let $\left\{p_{n}\right\}_{n \in \mathbb{N}}$ and $\left\{q_{n}\right\}_{n \in \mathbb{N}}$ be two strongly $\mathscr{I}$-statistically $D$-bounded sequences in the $\mathrm{PN}$ space $\left(X, \eta, \tau, \tau^{*}\right)$. Then, 
there exist sets $K=\left\{k_{1}<k_{2}<\cdots\right\} \subset \mathbb{N}$ and $L=\left\{l_{1}<\right.$ $\left.l_{2}<\cdots\right\} \subset \mathbb{N}$ such that for any $\delta>0,\{n \in \mathbb{N}:(1 / n) \mid\{k \leq$ $n: k \notin K\} \mid \geq \delta / 2\} \in \mathscr{I}$ and $\{n \in \mathbb{N}:(1 / n) \mid\{k \leq n: k \notin$ $L\} \mid \geq \delta / 2\} \in \mathscr{I}$ and $\mathscr{R}_{\{p\}_{K}}, \mathscr{R}_{\{q\}_{L}} \in \mathscr{D}^{+}$. Now, consider the set $K \cap L=\left\{n_{1}<n_{2}<\cdots\right\} \subset \mathbb{N}$. Obviously, $K \cap L \neq \emptyset$. We also have $\{n \in \mathbb{N}:(1 / n)|\{k \leq n: k \notin K \cap L\}| \geq$ $\delta\} \subseteq\{n \in \mathbb{N}:(1 / n)|\{k \leq n: k \notin K\}| \geq \delta / 2\} \cup\{n \in$ $\mathbb{N}:(1 / n)|\{k \leq n: k \notin L\}| \geq \delta / 2\}$. Since both sets on the right-hand side belong to $\mathscr{I}$, then $\{n \in \mathbb{N}:(1 / n) \mid\{k \leq n$ : $k \notin K \cap L\} \mid \geq \delta\} \in \mathscr{I}$. We observe that, for $n_{k} \in K \cap L$, $N_{p_{n_{k}}+q_{n_{k}}} \geq \tau\left(N_{p_{n_{k}}}, N_{q_{n_{k}}}\right) \geq \tau\left(N_{p_{n_{k}}}, \mathscr{R}_{\{q\}_{L}}\right) \geq \tau\left(\mathscr{R}_{\{p\}_{K}}, \mathscr{R}_{\{q\}_{L}}\right)$ and therefore we can write $\mathscr{R}_{\left\{p_{n_{k}}+q_{n_{k}}: k \in \mathbb{N}\right\}} \geq \tau\left(\mathscr{R}_{\{p\}_{K}}, \mathscr{R}_{\{q\}_{L}}\right)$, where $\mathscr{R}_{\{p\}_{K}} \in \mathscr{D}^{+}$and $\mathscr{R}_{\{q\}_{L}} \in \mathscr{D}^{+}$. By our assumption $\tau\left(\mathscr{R}_{\{p\}_{K}}, \mathscr{R}_{\{q\}_{L}}\right) \in \mathscr{D}^{+}$. Thus, $\mathscr{R}_{\left\{p_{n_{k}}+q_{n_{k}}: k \in \mathbb{N}\right\}} \in \mathscr{D}^{+}$for all $n_{k} \in K \cap L$ and $\{n \in \mathbb{N}:(1 / n)|\{k \leq n: k \notin K \cap L\}| \geq \delta\} \in \mathscr{I}$ for all $\delta>0$. Thus, the sequence $\left\{p_{n}+q_{n}\right\}_{n \in \mathbb{N}}$ yields a $D$ bounded subset of $X$. This completes the proof.

Corollary 52. Let $\left(X, \eta, \tau, \tau^{*}\right)$ be a PN space. If $\mathscr{D}^{+}$is invariant under $\tau$, that is, $\tau\left(\mathscr{D}^{+} \times \mathscr{D}^{+}\right) \subseteq \mathscr{D}^{+}$, then the set of all strongly $\mathscr{I}$-statistically $D$-bounded sequences in $X$ forms a real linear space.

\section{Conflict of Interests}

The authors declare that there is no conflict of interests regarding the publication of this paper.

\section{Acknowledgment}

The authors are thankful to the Council of Scientific and Industrial Research, HRDG, India, for granting the research Project no. 25(0186)/10/EMR-II during the tenure of which this work was done.

\section{References}

[1] H. Fast, "Sur la convergence statistique," Colloquium Mathematicum, vol. 2, pp. 241-244, 1951.

[2] J. A. Fridy, "On statistical convergence," Analysis, vol. 5, no. 4, pp. 301-313, 1985.

[3] T. Šalát, "On statistically convergent sequences of real numbers," Mathematica Slovaca, vol. 30, no. 2, pp. 139-150, 1980.

[4] J. A. Fridy, "Statistical limit points," Proceedings of the American Mathematical Society, vol. 118, no. 4, pp. 1187-1192, 1993.

[5] E. Kolk, “The statistical convergence in Banach spaces," Tartu Ülikooli Toimetised, no. 928, pp. 41-52, 1991.

[6] P. Kostyrko, T. Šalát, and W. Wilczyński, “ $\mathscr{I}$-convergence," Real Analysis Exchange, vol. 26, no. 2, pp. 669-685, 2000/01.

[7] P. Das and S. Kr. Ghosal, "Some further results on $\mathscr{I}$-Cauchy sequences and condition (AP)," Computers \& Mathematics with Applications, vol. 59, no. 8, pp. 2597-2600, 2010.

[8] K. Dems, "On $\mathscr{I}$-Cauchy sequences," Real Analysis Exchange, vol. 30, no. 1, pp. 123-128, 2004/05.

[9] P. Kostyrko, M. Mačaj, T. Šalát, and M. Sleziak, “ $\mathscr{I}$-convergence and extremal $\mathscr{I}$-limit points," Mathematica Slovaca, vol. 55, no. 4, pp. 443-464, 2005.
[10] V. Kumar and B. Lafuerza-Guillén, "On ideal convergence of double sequences in probabilistic normed spaces," Acta Mathematica Sinica, vol. 28, no. 8, pp. 1689-1700, 2012.

[11] B. K. Lahiri and P. Das, "Further results on $\mathscr{I}$-limit superior and limit inferior," Mathematical Communications, vol. 8, no. 2, pp. 151-156, 2003.

[12] B. K. Lahiri and P. Das, " $\mathscr{I}$ and $\mathscr{I}^{*}$-convergence in topological spaces," Mathematica Bohemica, vol. 130, no. 2, pp. 153-160, 2005.

[13] M. Mursaleen and S. A. Mohiuddine, "On ideal convergence in probabilistic normed spaces," Mathematica Slovaca, vol. 62, no. 1, pp. 49-62, 2012.

[14] A. Nabiev, S. Pehlivan, and M. Gürdal, "On $\mathscr{I}$-Cauchy sequences," Taiwanese Journal of Mathematics, vol. 11, no. 2, pp. 569-576, 2007.

[15] E. Savas, P. Das, and S. Dutta, "A note on strong matrix summability via ideals," Applied Mathematics Letters, vol. 25, no. 4, pp. 733-738, 2012.

[16] V. Karakaya, N. Şimşek, M. Ertürk, and F. Gürsoy, "Statistical convergence of sequences of functions in intuitionistic fuzzy normed spaces," Abstract and Applied Analysis, vol. 2012, Article ID 157467, 19 pages, 2012.

[17] V. Karakaya, N. Şimşek, M. Ertürk, and F. Gürsoy, “ $\lambda$-statistical convergence of sequences of functions in intuitionistic fuzzy normed spaces," Journal of Function Spaces and Applications, vol. 2012, Article ID 926193, 14 pages, 2012.

[18] P. Das, E. Savas, and S. Kr. Ghosal, "On generalizations of certain summability methods using ideals," Applied Mathematics Letters, vol. 24, no. 9, pp. 1509-1514, 2011.

[19] E. Savas and P. Das, "A generalized statistical convergence via ideals," Applied Mathematics Letters, vol. 24, no. 6, pp. 826-830, 2011.

[20] C. Belen and M. Yildirim, "On generalized statistical convergence of double sequences via ideals," Annali dell'Universitá di Ferrara, Sezione VII: Scienze Matematiche, vol. 58, no. 1, pp. 1120, 2012.

[21] K. Menger, "Statistical metrics," Proceedings of the National Academy of Sciences of the United States of America, vol. 28, pp. 535-537, 1942.

[22] B. Schweizer and A. Sklar, Probabilistic Metric Spaces, NorthHolland Publishing Co., New York, NY, USA, 1983.

[23] C. Sempi, "Probabilistic metric spaces", in Encyclopedia of General Topology, K. P. Hart, J. Nagata, and J. E. Vaughan, Eds., pp. 288-292, 2004.

[24] C. Alsina, B. Schweizer, and A. Sklar, "On the definition of a probabilistic normed space," Aequationes Mathematicae, vol. 46, no. 1-2, pp. 91-98, 1993.

[25] C. Alsina, B. Schweizer, and A. Sklar, "Continuity properties of probabilistic norms," Journal of Mathematical Analysis and Applications, vol. 208, no. 2, pp. 446-452, 1997.

[26] B. Lafuerza-Guillén, "Finite products of probabilistic normed spaces," Radovi Matematički, vol. 13, no. 1, pp. 111-117, 2004.

[27] B. Lafuerza-Guillén, "D-bounded sets in probabilistic normed spaces and in their products," Rendiconti di Matematica e delle sue Applicazioni, vol. 21, no. 1-4, pp. 17-28, 2001.

[28] B. Lafuerza Guillén, J. A. Rodríguez Lallena, and C. Sempi, "Probabilistic norms for linear operators," Journal of Mathematical Analysis and Applications, vol. 220, no. 2, pp. 462-476, 1998.

[29] B. Lafuerza Guillén, J. A. Rodríguez Lallena, and C. Sempi, "A study of boundedness in probabilistic normed spaces," Journal 
of Mathematical Analysis and Applications, vol. 232, no. 1, pp. 183-196, 1999.

[30] B. Lafuerza Guillén, J. A. Rodríguez Lallena, and C. Sempi, "Some classes of probabilistic normed spaces," Rendiconti di Matematica e delle sue Applicazioni, vol. 17, no. 2, pp. 237-252, 1997.

[31] B. Schweizer and A. Sklar, "Statistical metric spaces," Pacific Journal of Mathematics, vol. 10, pp. 313-334, 1960.

[32] B. Schweizer and A. Sklar, "Statistical metric spaces arising from sets of random variables in Euclidean $n$-space," Theory of Probability and Its Applications, vol. 7, pp. 456-465, 1962.

[33] B. Schweizer and A. Sklar, "Triangle inequalities in a class of statistical metric spaces," Journal of the London Mathematical Society, vol. 38, pp. 401-406, 1963.

[34] B. Schweizer, A. Sklar, and E. Thorp, "The metrization of statistical metric spaces," Pacific Journal of Mathematics, vol. 10, pp. 673-675, 1960.

[35] R. M. Tardiff, "Topologies for probabilistic metric spaces," Pacific Journal of Mathematics, vol. 65, no. 1, pp. 233-251, 1976.

[36] C. Şençimen and S. Pehlivan, "Strong statistical convergence in probabilistic metric spaces," Stochastic Analysis and Applications, vol. 26, no. 3, pp. 651-664, 2008.

[37] C. Şençimen and S. Pehlivan, "Statistical continuity in probabilistic normed spaces," Applicable Analysis, vol. 87, no. 3, pp. 377-384, 2008.

[38] C. Şençimen and S. Pehlivan, "Statistically D-bounded sequences in probabilistic normed spaces," Applicable Analysis, vol. 88, no. 8, pp. 1133-1142, 2009.

[39] C. Şençimen and S. Pehlivan, "Strong ideal convergence in probabilistic metric spaces," Proceedings of the Indian Academy of Sciences: Mathematical Sciences, vol. 119, no. 3, pp. 401-410, 2009.

[40] S. A. Mohiuddine and E. Savaş, "Lacunary statistically convergent double sequences in probabilistic normed spaces," Annali dell'Universitá di Ferrara, Sezione VII: Scienze Matematiche, vol. 58, no. 2, pp. 331-339, 2012.

[41] P. Das, K. Dutta, V. Karakaya, and S. Ghosal, "On some further generalizations of strong convergence in probabilistic metric spaces using ideals," Abstract and Applied Analysis, vol. 2013, Article ID 765060, 8 pages, 2013.

[42] C. Şençimen and S. Pehlivan, "Probabilistic normed Riesz spaces," Acta Mathematica Sinica, vol. 28, no. 7, pp. 1401-1410, 2012. 


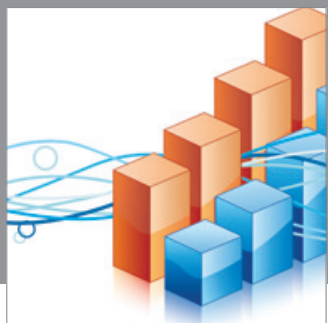

Advances in

Operations Research

mansans

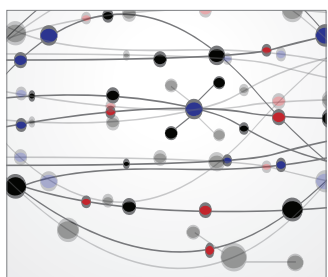

The Scientific World Journal
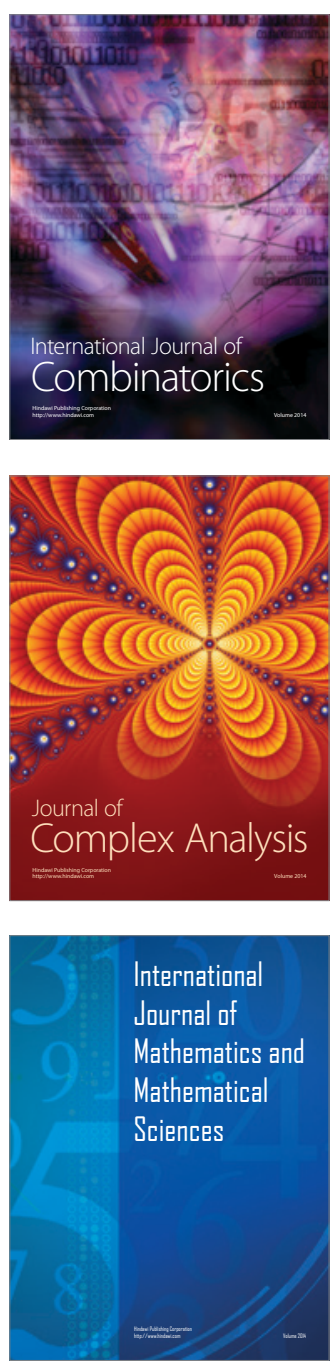
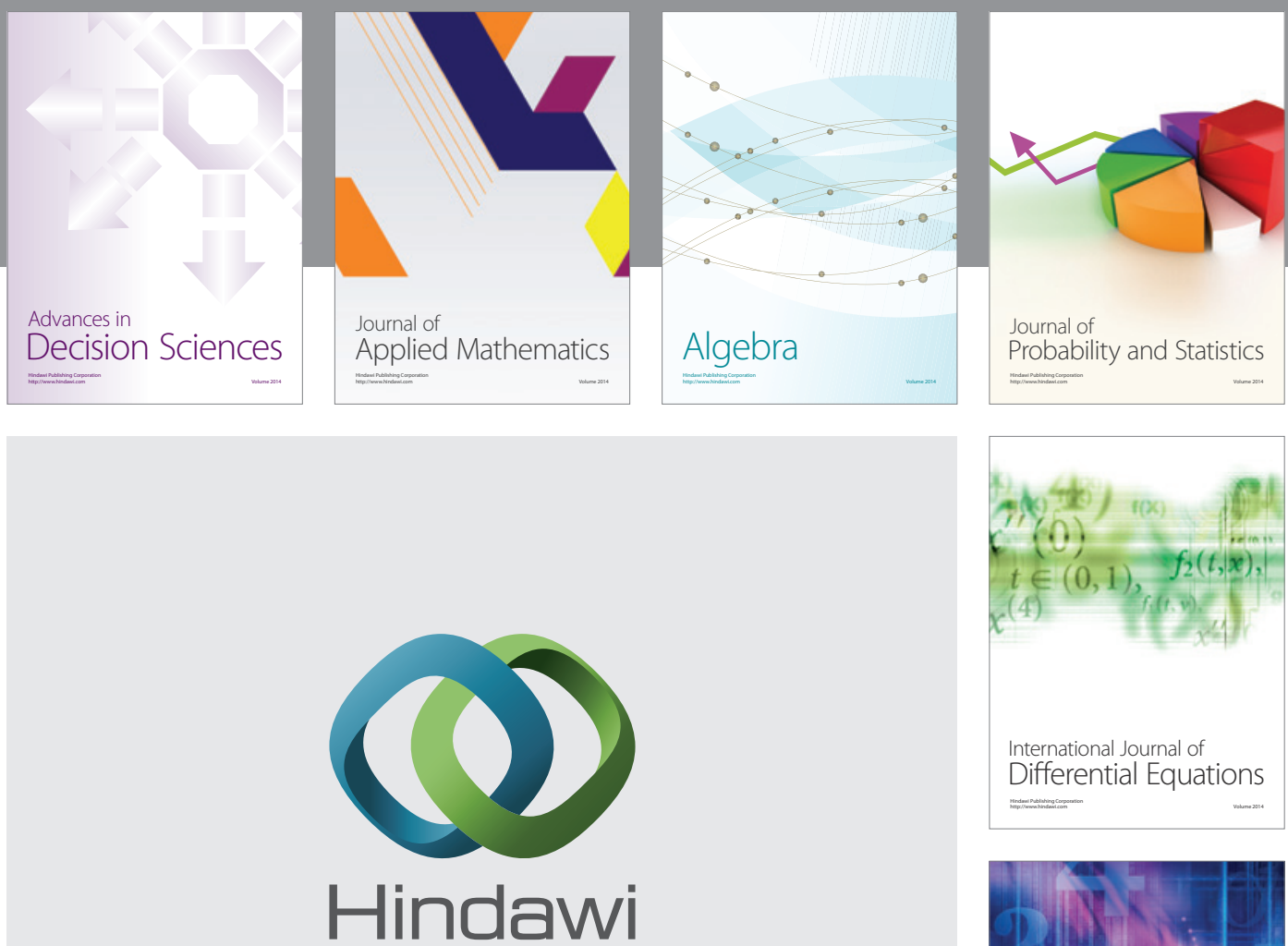

Submit your manuscripts at http://www.hindawi.com
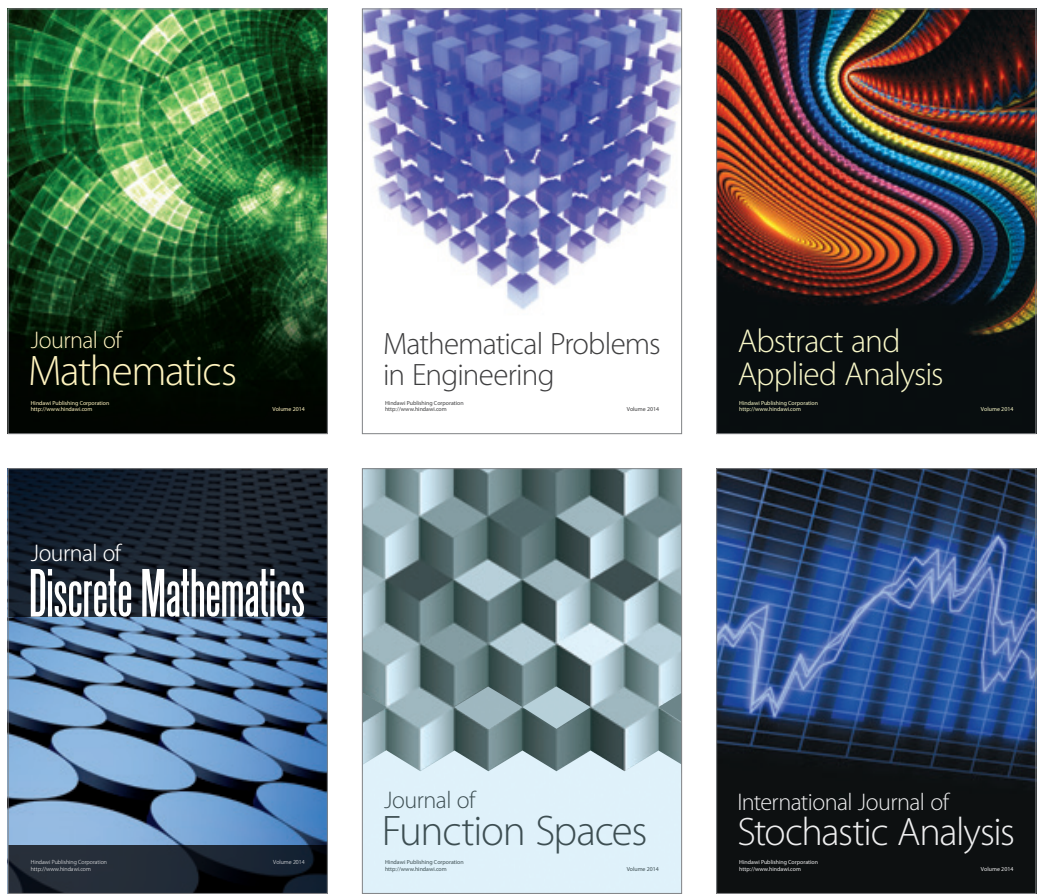

Journal of

Function Spaces

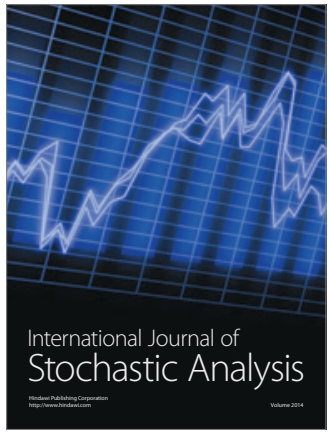

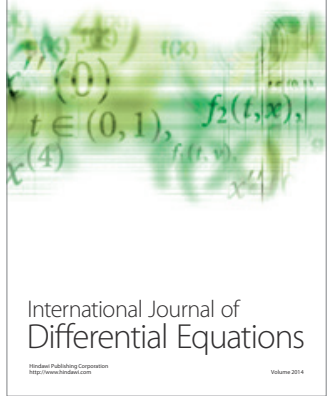
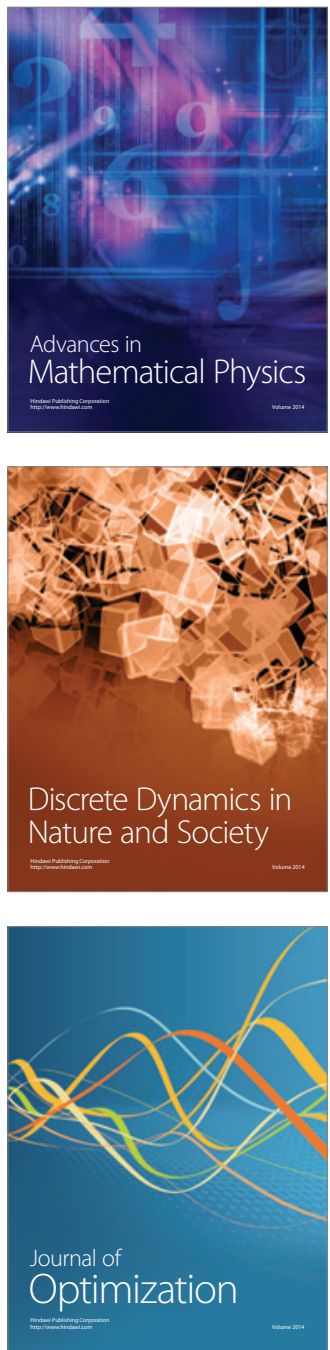